\title{
El rol de las redes sociales para futuros periodistas. Manejo, uso y comportamiento de estudiantes y profesores universitarios de Ecuador
}

\section{The role of social networks for future journalists. Management, use and behavior of students and university professors of Ecuador}

\section{O papel das redes sociais para futuros jornalistas. Manejo, uso e comportamento de estudantes e professores universitários de Equador}

Raquel Martínez-Sanz, Universidad de Valladolid, Valladolid, España (raquel.martinez.sanz@uva.es)

Amaia Arribas-Urrutia, Universidad de Los Hemisferios, Quito, Ecuador (amayaa@uhemisferios.edu.ec)

RESUMEN | Las redes sociales cambian las rutinas productivas, de difusión y de consumo de los medios de comunicación. Los futuros periodistas y sus docentes se enfrentan al desafío de su uso profesional y personal. Esta investigación indaga en los usos, preferencias de consumo y dominio de las redes sociales de estos dos colectivos en varias universidades ecuatorianas. La triangulación metodológica (encuesta, focus group y panel de expertos) aborda la relación que establecen con las redes sociales, el efecto en su día a día y en el quehacer docente. Los resultados revelan que los estudiantes tienen un perfil activo en redes sociales. Usan principalmente el smartphone para visualizar, compartir y crear contenido para entretenerse e informarse y manifiestan interés por formar parte de una comunidad de usuarios. También consideran a las redes sociales como un escaparate profesional y sienten necesidad de cuidar sus espacios digitales. Los docentes ven en las redes sociales un lugar para actualizarse y siguen a profesionales o medios de comunicación de confianza. Su presencia en las redes es limitada, caracterizada por la observación, y no se sienten lo suficientemente formados en tecnologías para su práctica docente, ni que disponen de tiempo para ello.

PALABRAS CLAVE: redes sociales; comunicación; educación superior; periodismo; convergencia mediática; smartphone. 
ABSTRACT | Social networks has led to a change in the media production, dissemination, and consumption routines. Futurejournalists and their professors face the challenge of their professional and personal use. This research investigates the uses, consumption preferences, and capacity of control of these two groups regarding social networks in different universities in Ecuador. The methodological triangulation (survey, focus group and panel of experts) allows to observe the relationship that communication students and professors have with social networks and the effect on their teaching work. The results reveal that young university students have an active profile on social networks where, mainly through the smartphone, they view, share, and create content to be entertained and informed, expressing interest in being part of a community of users. Likewise, they consider social networks as a professional showcase and feel the need to take care of their digital spaces. Professors see social media as a place to stay updated, following trusted professionals or media. Their presence in social networks is limited, characterized by observation, and they do not feel sufficiently trained in technologies for their teaching practice, nor they have the time to do it.

KEYWORDS: social networks; communication; higher education; journalism; media convergence; smartphone.

RESUMO|As redes sociais mudam as rotinas produtivas, de difusão e de consumo da mídia. Os futuros jornalistas e seus docentes enfrentam o desafio de seu uso profissional e pessoal. Essa pesquisa indaga nos usos, as preferências de consumo e domínio das redes sociais destes dois grupos em várias universidades equatorianas. A triangulação metodológica (questionário, focus group e painel de especialistas) aborda a relação estabelecida com as redes sociais, o efeito no seu dia a dia e no trabalho docente. Os resultados mostram que os estudantes têm um perfil ativo nas redes sociais. Eles usam principalmente o smartphone para visualizar, compartilhar e criar conteúdo para se divertirem e informarem, e expressam interesse em fazer parte de uma comunidade de usuários. Eles também consideram as redes sociais como uma vitrine profissional e sentem a necessidade de cuidar de seus espaços digitais. Os professores veem nas redes sociais um lugar para se atualizarem e seguirem a profissionais ou mídia de confiança. Sua presença nas redes élimitada, caracterizada pela observação, e eles não sentem que estão suficientemente treinados em tecnologias para sua prática de ensino, nem que têm tempo para fazê-lo.

PALAVRAS-CHAVE: redes sociais; comunicação; educação superior; jornalismo; convergência de mídia; smartphone. 


\section{INTRODUCCIÓN}

Ecuador es uno de los países latinoamericanos que presenta un mayor índice de penetración de Internet (We are social \& Hootsuite, 2018; Internet World Stats, 2019), especialmente entre los jóvenes, llegando al 97\% según el World Internet Project Ecuador (Arribas-Urrutia, Islas-Carmona, \& Gutiérrez, 2019). La rápida expansión de las redes sociales está contribuyendo a normalizar su uso en ámbitos tan dispares como la educación (García-Ruiz, Gozálvez, \& Aguaded, 2014; Martínez-Sanz, Islas Carmona, Campos Domínguez, \& Redondo García, 2016), la gobernanza (Caridad \& Martínez, 2016; Negrete-Huelga \& Rivera-Magos, 2018) o el sector empresarial (Durántez-Stolle, 2017), fuertemente condicionados por el ecosistema digital.

Esta irrupción ha transformado la forma de entender, enseñar y ejercer el periodismo (García Orosa \& López García, 2016), dando lugar a una convergencia mediática que refuerza las posibilidades de acción y de participación delas audiencias (Jenkins, 2008). Este paradigma trae consigo un cambio narrativo, organizativo y de modelo de negocio en el que las redes sociales desempeñan un rol polinizador y de valor agregado, sujeto a la capacidad de gestionar y crear comunidad (CamposFreire, Rúas-Araújo, López-García, \& Martínez-Fernández, 2016).

Por otra parte, la actividad docente también se ha visto afectada, tanto desde el punto de vista del proceso de enseñanza y aprendizaje (Barrios \& Fajardo, 2017) como por la incorporación de materias en los planes de estudios relacionadas con el periodismo multimedia, el periodismo inmersivo y el periodismo de datos (López-García, Rodríguez-Vázquez, \& Pereira-Fariña, 2017; Chaparro Domínguez, 2014). Tejedor y Cervi (2017) subrayan que, para continuar dotando de relevancia a la profesión, es preciso actualizar los programas curriculares acorde con las competencias y perfiles demandados por el mercado laboral (Lopezosa, Codina, Díaz-Noci, \& Ontalba, 2020), y entregar una formación tecnológica al profesorado.

La sobreabundancia de información, en ciertas ocasiones tendenciosa, el interés por influir en la audiencia o la recopilación de datos derivados de nuestras acciones virtuales son solo algunos de los peligros asociados a la consulta de Internet (Caro Samada, 2015) y a los que los futuros periodistas y sus profesores se enfrentan a diario. Este trabajo, además de indagar en los hábitos de consumo informativo, tiene como objetivo secundario conocer la percepción y rutinas de interacción en las redes sociales de estos dos colectivos llamados a entenderse, lo que representa el principal reto de esta investigación. El uso de las redes sociales es parte de la evolución de la profesión periodística que será ejercida por quienes ahora se están formando, e investigar en esta área puede contribuir a fundamentar futuros cambios en el planteamiento de los estudios de esta profesión, así como en las metodologías de su enseñanza. 


\section{MARCO TEÓRICO}

\section{Internet en la profesión y enseñanza del periodismo}

El devenir de la profesión periodística y su enseñanza atraviesa un momento crucial con Internet y, sobre todo, con las redes sociales. En apenas dos décadas, se han multiplicado los medios digitales y las rutinas periodísticas han cambiado radicalmente (Mayoral Sánchez, 2016; Navarro Zamora, 2012). La multiplicidad de plataformas ha provocado profundos cambios en el mapa mediático, afectando a los medios convencionales sin excepción. Asimismo, se atomizan los roles ligados a la producción y al consumo de información gracias a la movilidad y a la personalización de los contenidos suministrados por los medios, y a la inclusión de las redes sociales dentro de su estructura de difusión y de relación con las audiencias. Por ello, el análisis de la Web 2.0 es de vital importancia para comprender el funcionamiento y estructura de las instituciones y la relación que mantienen con el periodismo y con las diferentes formas de comunicación (Sampedro, 2015), ya que son los individuos los que conforman esas redes sociales y quienes, en definitiva, logran los cambios y las transformaciones sociales.

Sin embargo, para Ramón Salaverría (2010), el despegue definitivo de estos medios digitales aún no se producía principalmente por dos razones: la ausencia de un auténtico modelo de negocio, a pesar de las enormes audiencias en Internet, y la carencia de la innovación en cuanto a su estructura y lógica de contenidos que replican lo que está en los medios tradicionales. El público elige informarse mediante plataformas digitales, mientras que los grandes grupos mediáticos se ven castigados por la baja inversión publicitaria que ha migrado a estos espacios (Martínez-Costa, Serrano-Puche, Portilla, \& Sánchez-Blanco, 2019). Los consejos que Salaverría propuso en su momento para afrontar este incierto panorama siguen vigentes (Túñez-López, Fieiras Ceide, \& Vaz-Álvarez, 2021), y se dirigen hacia la apuesta por el reporteo en Internet, la innovación en géneros y formatos, la elaboración de libros de estilo para los cibermedios y la renovación del currículum formativo de los estudios de periodismo en la universidad, entre otras ideas.

Respecto de la necesidad de actualizar los contenidos curriculares, se busca una coherencia con los perfiles laborales más demandados vinculados con la transformación digital. Así, se deben regenerar los planes de estudio para formar a los profesionales del periodismo hacia un ecosistema mediático digital que poco a poco resulta un hecho en las universidades latinoamericanas (Cuesta \& Lugo-Ortiz, 2017; Mellado, 2011). Sin embargo, más allá de incorporar asignaturas relacionadas con Internet "debemos enseñar a practicarlo con, en y sobre y mediante Internet [...] solo los periodistas que dominen a fondo los fundamentos de la comunicación digital estarán en condiciones de construir en la red los cimientos de una profesión periodística renovada" (Salaverría, 2010, p. 248). 
En este mismo sentido, podemos señalar que el cambio curricular debe ir encaminado hacia un ciberperiodismo que integre tanto las prácticas tradicionales como las emergentes, sin formar necesariamente un profesional tecnológicamente preparado, sino que conciba a las tecnologías como una herramienta que facilita su objetivo como periodista, que es el de buscar democratizar la información (Nieto Borda, 2021).

La formación del profesorado en competencias tecnológicas es un tema ampliamente investigado (Sandí Delgado \& Sanz, 2018; Martínez-Sanz et al., 2016). Sin embargo, conviene remarcar que la vertiente digital forma parte de la competencia profesional de los docentes de cualquier nivel de enseñanza; por lo tanto, no solo se trata de dotarlos de equipamiento tecnológico para la docencia, sino de una capacitación constante y actualizada en dos sentidos: el manejo de los dispositivos digitales y la pedagogía adecuada para un proceso de enseñanza-aprendizaje productivo (Durán Cuartero, Gutiérrez Porlán, \& Prendes Espinosa, 2016). Celia Castaño (2016) defiende que el docente debe saber utilizar la tecnología desde diferentes perspectivas: técnica, aplicada, social y ética. De igual manera, Shapiro y Hudhese (1996, en Bawden, 2002) describen hasta siete dimensiones en torno a esta alfabetización digital orientada hacia el profesorado que contempla aspectos como:

- La adquisición de destrezas en el uso de herramientas vinculadas con la tecnología de la información;

- El dominio de las formas y métodos de acceso a los recursos web;

- La comprensión del contexto social y de la producción de la información;

- La capacidad para aplicar el uso de las herramientas tecnológicas a la investigación y al trabajo académico;

- La habilidad para difundir información;

- La visión y comprensión de conjunto de las innovaciones tecnológicas que le permita tomar las mejores decisiones en torno a ellas;

- La capacidad para evaluar de manera crítica los beneficios y costos de las tecnologías, lo que supone incorporar una vertiente ética a dicha alfabetización.

Los profesores de periodismo y sus estudiantes se enfrentan al uso de Internet y de las redes sociales como herramientas difusoras de información. No obstante, para los medios de comunicación proporcionar información ya no es suficiente, sino que tienen que ofrecer espacios de comunicación y socialización con sus 
públicos. Como afirma Lara (2008), no basta con producir y ofrecer noticias: ahora los medios tienen que dar servicio y ser útiles a sus audiencias, propiciando una participación abierta y de calidad.

[...] es una forma de reconectar con la sociedad y superar las crisis de autoridad, mediación y credibilidad en la que está sumergida actualmente la prensa. Ante este panorama, los medios necesitan crear contextos donde sus audiencias puedan interactuar con el medio y con otros usuarios, donde la gente sienta el espacio como suyo, como un lugar de pertenencia y de referencia personal y comunitaria (Lara, 2008, p. 129).

Para Noguera (2010), la mayor potencialidad de las redes sociales está en la conversión del periodismo hacia un modelo más social y transparente, con una arquitectura para la gestión de la última hora, la búsqueda de nuevas fuentes y la constante contextualización de los contenidos en espacios más sociales y dinámicos que los que los cibermedios ofrecen. En este sentido, el autor señala que "las redes, con su comportamiento y sus audiencias, han convencido a los medios de que no pueden alcanzar a nuevos públicos si no renuncian a la equivocada premisa de mantener al lector, literalmente, bajo sus dominios en la Web" (p. 181).

El uso de las redes sociales por parte de los periodistas es un factor clave para la distribución y el consumo de noticias. Estos espacios digitales contribuyen a la revalorización de sus autores, siempre y cuando exista una gestión planificada y una adaptación de los contenidos a la idiosincrasia de la plataforma y a las demandas de sus audiencias (Picard, 2014). The Washington Post ya advertía en su manual de uso al periodista que no olvidara su condición de representante del medio durante sus apariciones por redes sociales, y que la precisión, junto con la transparencia eran requisitos en sus intervenciones. Tascón rescata las guías que The Washington Post dio a sus periodistas para el uso de redes sociales: "Debemos recordar que los periodistas del Washington Post somos siempre periodistas del Washington Post" (2012, p. 86). A partir de esta declaración de intenciones, queda claro que para el medio estadounidense hasta los perfiles personales deben atenerse a las normas de la empresa, limitando cualquier uso privado de este espacio.

Con las redes sociales, el periodista ha visto favorecida la visibilidad de su trabajo. Si este profesional tiene la competencia y la habilidad para gestionar su marca personal podrá crear un valor agregado en su credibilidad como fuente informativa. Asimismo, el entorno digital presenta una gran oportunidad para emprender proyectos periodísticos innovadores, proporcionando autonomía a los periodistas. Noguera señala que 


\section{La gestión de la identidad digital debería estar en el primer lugar en la lista de prioridades. Sin un correcto análisis de quiénes somos para quienes somos un desconocido, es decir, del perfil que ofrecemos en la web sobre nuestra identidad, difícilmente puede emprenderse con éxito cualquier tipo de pro- yecto personal digital (2012, p. 15).}

En el caso de Ecuador, todavía hay mucho camino por recorrer en cuanto a la creación de una marca personal digital de los periodistas ecuatorianos, si bien se observa un crecimiento continuo en la credibilidad de los cibermedios (OdriozolaChéné \& Rodrigo-Mendizábal, 2017). Los investigadores Yáñez, Martínez y Barrios (2018) señalan que los periodistas ecuatorianos han logrado su prestigio en medios de comunicación tradicionales sin recurrir a las redes sociales para fortalecer su identidad. Añaden que los periodistas ecuatorianos no han mostrado demasiado interés en la construcción de una marca personal digital, y que su reconocimiento viene dado por el programa que presentan o por el medio de comunicación para el que trabajan. Según los autores, son todavía muy pocos los profesionales que apuestan por gestionar su identidad en el ciberespacio apoyándose en las redes sociales.

\section{METOdOLOGÍA}

Este trabajo es de tipo descriptivo y se centra en analizar cómo participan en las redes sociales los futuros periodistas y su profesorado en varias universidades de Ecuador, para identificar cómo interactúan con el contenido informativo dispuesto en la red, ahondando en las competencias digitales de las que disponen, el equipamiento tecnológico empleado y la predisposición para generar contenido.

Se formula una hipótesis en relación con cada uno de los colectivos estudiados:

H1. El alumnado participa en redes sociales de forma impulsiva, proactiva y multipantalla, condicionado, en buena parte, por su predisposición hacia la experimentación en el uso de las tecnologías de la información y de las comunicaciones (TIC), y que aplica tanto a su vertiente personal como preprofesional. Estudios previos como el de Herrero-Diz, Tapia-Frade y Varona-Aramburu (2020) demuestran la consolidación de los dispositivos móviles para el consumo de información.

H2. La incursión digital del docente en las redes sociales se rige por la cautela, la observación y la sistematización de acciones. Distintas organizaciones internacionales inciden en la importancia de la formación del profesorado en las TIC como un factor clave para la innovación y la mejora educativa; sin embargo, trabajos como los de García-Pérez, Rebollo-Catalán y GarcíaPérez (2016) demuestran un uso residual de las redes sociales por parte del profesorado como herramienta docente. 
Para asegurar la fiabilidad de los datos, esta investigación recurrió a la triangulación metodológica, que permite el abordaje, desde diferentes métodos y técnicas, de un mismo objeto de estudio. Según Morse (1991), la triangulación metodológica implica el uso de al menos dos métodos, normalmente uno cuantitativo y uno cualitativo, para estudiar el mismo problema de investigación. La triangulación metodológica se realizó dentro del método -within method- y entre métodos -across method- (Arias, 2000). La primera consistió en la recolección de datos de diferentes sujetos (expertos y encuestados) para medirlas mismas variables. La segunda se apoyó en la combinación de diferentes técnicas (grupos focaless, entrevista en profundidad y encuestas) para asegurar la aproximación al fenómeno objeto de estudio.

De forma más específica, la aproximación al colectivo de los jóvenes universitarios se hizo mediante una encuesta en línea y tres grupos de discusión sobre estudiantes de pregrado y maestría en Comunicación de la Universidad de Los Hemisferios (UHemisferios) en Quito, Ecuador. Todo ello con la finalidad de conocer, mediante testimonio directo, aspectos como el perfil que los futuros periodistas adoptan en redes sociales y el uso informativo que realizan; cómo y en qué circunstancias divulgan contenidos, o qué rol desempeñan los soportes móviles en esos procesos.

La población total de pregrado y posgrado en Comunicación es de 150 alumnos $(\mathrm{N}=150)$. La encuesta fue respondida por 51 alumnos de pregrado y 10 de posgrado $(n=61)$, lo que supone un margen de error de 5,8 y un nivel de confianza de $95 \%$, considerando además que no existe una máxima indeterminación ya que en la muestra está más representada el perfil de pregrado $\mathrm{p}(90)$ que el de posgrado q(10). El cuestionario, aplicado a una muestra no probabilística accesible, contó con siete preguntas específicas dedicadas al tema que aborda este estudio, y fue distribuido en línea entre los estudiantes de los cuatro cursos que integran el pregrado y la maestría en Comunicación de la UHemisferios (ver figural). Los grupos de discusión, por el contrario, se desarrollaron de forma presencial en tres grupos de ocho integrantes y se prolongaron, en promedio, 40 minutos, en las instalaciones de la universidad.

Para obtener la visión del otro colectivo implicado, el de los docentes, se recurrió al método Delphi: se constituyó un panel de 18 expertos - 9 hombres y 9 mujeres-, todos ellos reputados profesores de Comunicación en cuatro universidades ecuatorianas: Universidad de Los Hemisferios, Universidad Técnica Particular de Loja, Universidad de las Américas y Universidad Andina Simón Bolívar, a quienes se les hizo llegar un cuestionario de 22 preguntas ordenadas por bloques temáticos: 1. Equipamiento tecnológico y disposición a continuar formándose en su manejo; 2. Las plataformas digitales como vehículo de información; 3. Fiabilidad y espectacularización de los contenidos en la Web, y 4. Tipos de participación a través de las redes sociales. 


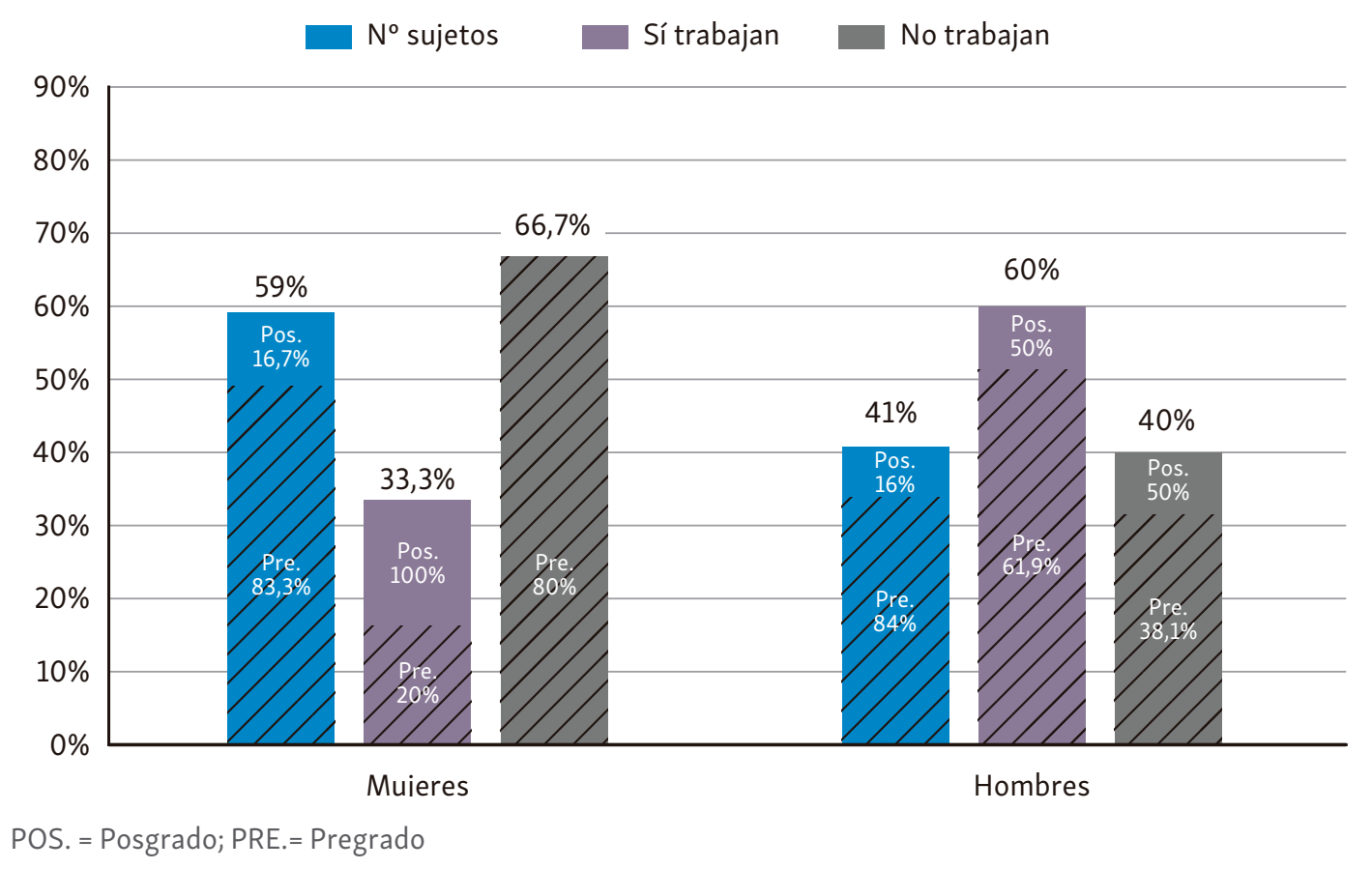

Figura 1. Rasgos sociodemográficos de la muestra de estudiantes encuestados

Fuente: Elaboración propia.

Los datos obtenidos mediante las encuestas fueron codificados a través del programa estadístico SPSS, mientras que los grupos de discusión y las entrevistas en profundidad fueron grabados y transcritos.

\section{RESULTADOS}

Un alumnado que apuesta por la volatilidad y se recrea en la observación

Los jóvenes universitarios encuestados $(\mathrm{n}=61)$ tienen un perfil activo en una media de 3,65 redes sociales. Tal y como muestra la figura 2 , casi la totalidad de la muestra está presente en Facebook, aunque cuando se trata de señalar cuál de todas es su favorita, un tercio se decanta por Facebook (37,7\%), otro tercio marca Instagram $(36,1 \%)$, y el restante se fragmenta en el resto de las opciones.

Sin embargo, si nos fijamos en el género de los encuestados $-41 \%$ son hombres y 59\%, mujeres-, observamos que la preferencia de estas últimas por Instagram $(41,7 \%)$ y Facebook $(38,9 \%)$ está por encima de la media, mientras que el número de perfiles abiertos y en uso coincide con el de los hombres $(3,6)$.

Los grupos de discusión sirvieron para comprobar que Snapchat, Instagram y Facebook fueron las aplicaciones más nombradas entre los participantes de edades comprendidas entre los 20 y los 25 años, mientras que aquellos que la sobrepasan -alumnos de posgrado, en su mayoría- vuelcan sus comentarios de preferencias y costumbres hacia Facebook, Twitter y LinkedIn. 


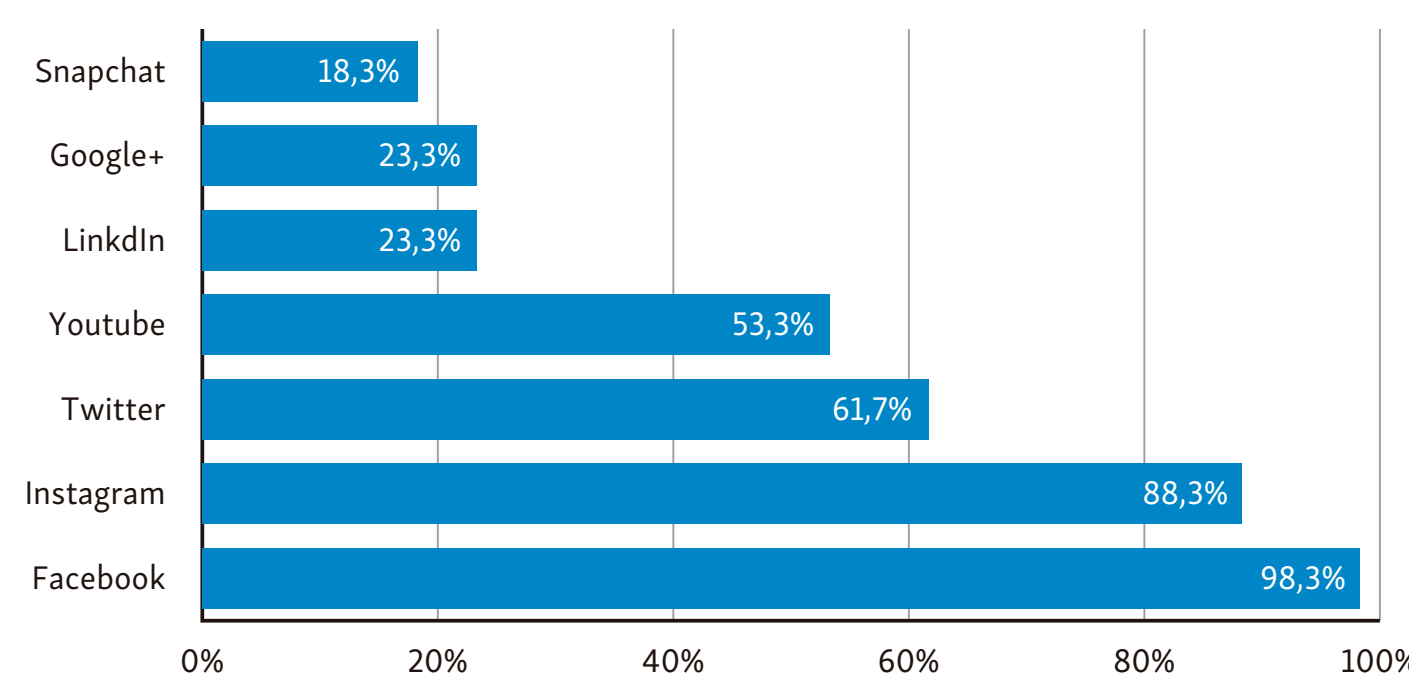

Figura 2. Porcentaje de jóvenes universitarios con cuenta actualizada

Fuente: Elaboración propia.

Más de la mitad de los estudiantes declaran utilizar su red social favorita prioritariamente para entretenerse $(67,2 \%)$, estar informados $(62,3 \%)$ y socializar $(55,7 \%)$. “Como estudiante de Comunicación, me interesa la información y a cada rato estoy viendo qué noticias hay" (hombre, 21 años). Otros usos referidos son como un canal de promoción profesional $(24,6 \%)$ o de activismo social (21,3\%).

Curiosamente, el afán de denuncia, de manifestarse cuando sienten que se ha producido una injusticia, es una motivación recurrente entre los jóvenes para publicar una entrada de autoría propia en sus espacios virtuales: el maltrato animal, el incivismo, la corrupción política o los desastres naturales fueron las cuestiones más señaladas durante la celebración de los grupos de discusión. Estos encuentros sirvieron para ratificar que la principal finalidad de acceder a redes sociales es el entretenimiento: ver lo que hacen los demás y estar pendientes de las últimas tendencias.

Independientemente de la intencionalidad perseguida, el smartphone es el soporte por excelencia para visualizar y crear contenido. La tableta y el ordenador son descartados como herramienta de acceso-nunca se utiliza- por un $59 \%$ y un $8,2 \%$, respectivamente. En esta línea se enmarcan las valoraciones recogidas durante los focus group: "El celular se ha acabado convirtiendo en un elemento imprescindible de nuestras vidas, incluso determinadas tareas escolares se resuelven con él” (mujer, 20 años). Asimismo, sienten que no pueden dejar su teléfono apartado: "Cuando estoy un tiempo largo sin mirarlo, cuando lo recupero está que explota" (mujer, 20 años). Algunos sienten que esa necesidad ha sido impuesta por un mercado laboral cada vez más exigente en relación con la conectividad: "Tengo que estar pegada al teléfono, mi trabajo, que está vinculado con la comunicación, me lo exige. Sé de personas que incluso lo han firmado por contrato" (mujer, 26 años). 
También existe coincidencia a la hora de adjudicarse la capacidad para poder desempeñar de forma eficiente varias tareas de forma simultánea (multitasking), siendo la consulta del celular la que siempre se combina con el resto: escuchar al profesor, seguir el hilo de una conversación o ver una película.

Algunos estudiantes entienden las redes sociales como un escaparate para proyectarse profesionalmente y sienten la obligación de cuidar sus espacios digitales: "No sabes quién te puede ver o qué oportunidad laboral se puede presentar" (hombre, 37 años). "Instagram hace que pueda plasmar mi personalidad tal y como es, y poco a poco crear una imagen visual de mi misma” (mujer, 22 años). Esa conciencia de la transcendencia que tienen sus acciones justifica en gran medida la utilización de aplicaciones que tienen como rasgo distintivo la volatilidad de los contenidos publicados. "Con Snapchat, los contenidos desaparecen a las 24 horas. Desaparece el riesgo de que alguien dentro de 5, 10 o 20 años saque una foto que eche por tierra tu reputación" (hombre, 21 años). Quieren proteger su intimidad. Sin embargo, incluso quienes se definen como usuarios pasivos reconocen publicar de forma esporádica aspectos de su vida privada. "¿La última vez que publiqué algo relativo a mi vida personal? ... Hace bastante, por lo menos 6-7 meses, por la muerte de mi papá" (hombre, 38 años).

El estudio longitudinal de Arribas-Urrutia, Islas-Carmona y Gutiérrez (2019) realizado a través del World Internet Project (WIP) reconoció la tendencia al alza de los jóvenes ecuatorianos por compartir y viralizar contenidos de otros. Nuestra encuesta, distribuida de forma específica entre universitarios, reafirma esta situación, pues $83,6 \%$ se conecta a sus perfiles en redes sociales diariamente con la finalidad de leer/visualizar los contenidos de otros, y 57,4\% lo hace a diario o varias veces a la semana para crear sus propias entradas (figura 3).

Al ser preguntados por cuáles habían sido las formas de interacción puestas en práctica en los últimos seis meses con alguna organización o marca, 38,5\% de los encuestados señaló retuitear o marcar como me gusta/no me gusta algún contenido. "Fácilmente, puedo dedicar 10 horas al día a las redes sociales. Compartir y ver lo que suben los demás me ocupa la mayor parte del tiempo" (mujer, 22 años).

En lo que respecta a utilizar las redes sociales para mantenerse informado, tan solo $21,8 \%$ declaró seguir a algún medio de comunicación o periodista destacado. "Me entero de ciertas noticias no porque las busque, sino porque me saltan" (mujer, 20 años), mientras que otros apuntan que, como futuros comunicólogos, sienten la necesidad de compartir y hacer llegar a sus círculos más cercanos las informaciones de mayor transcendencia. Llama la atención que durante la celebración de los grupos de discusión los jóvenes entre 20 y 25 años manifestaron gran interés por formar parte de la comunidad de usuarios de ciertos influencers -instagramers o YouTubers-, 


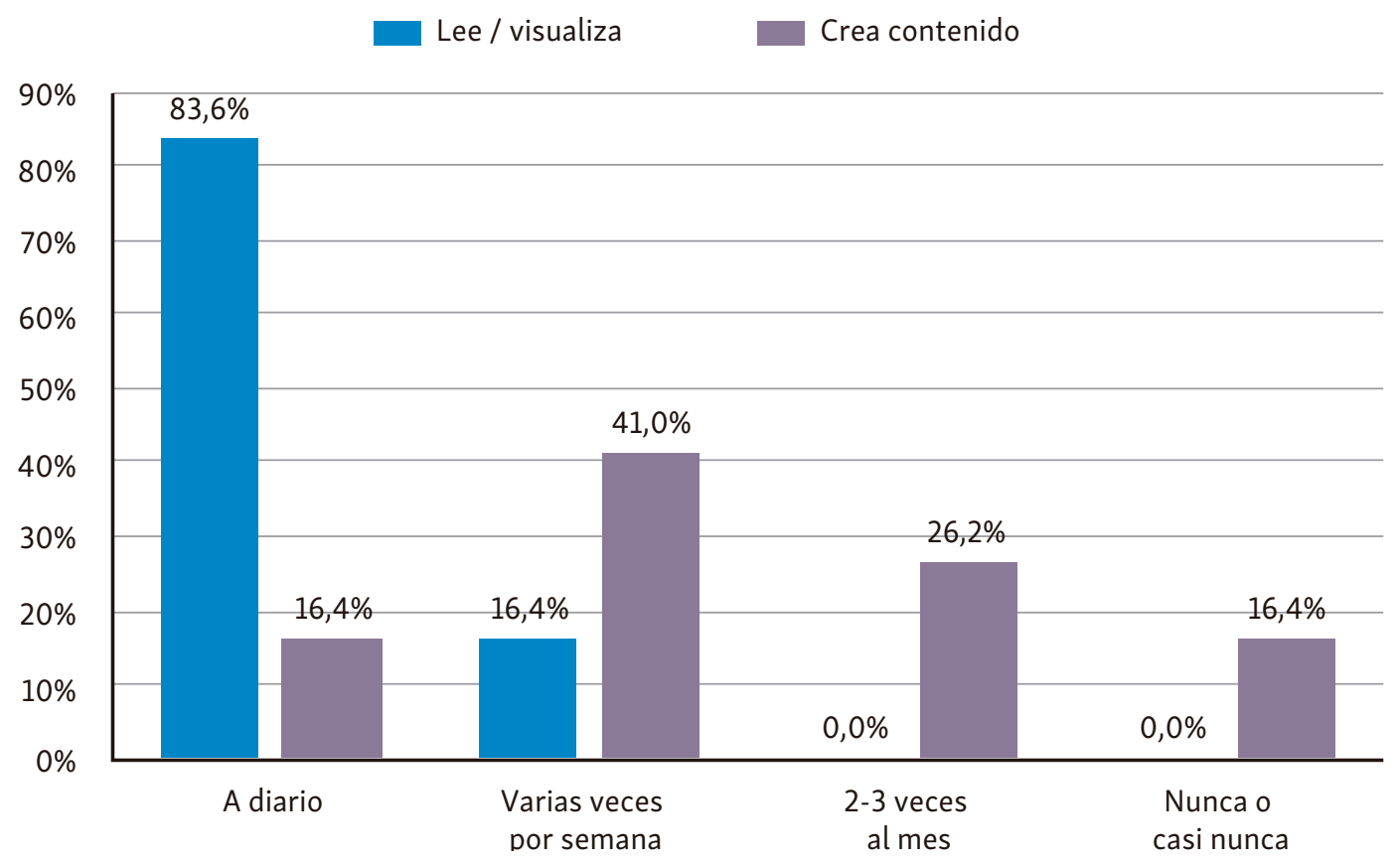

Figura 3. Frecuencia e intencionalidad en el acceso a las redes sociales

Fuente: Elaboración propia.

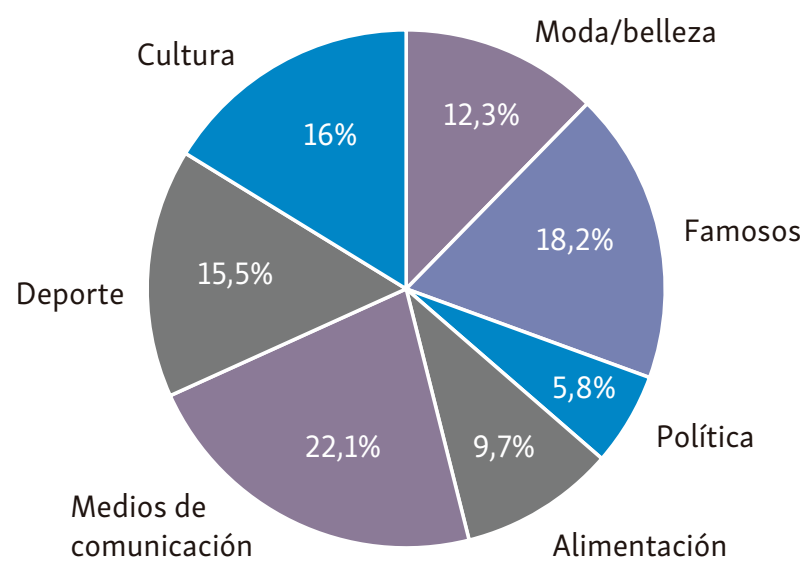

Figura 4. Sector al que pertenecen las organizaciones a las que siguen los universitarios a través de las redes sociales

Fuente: Elaboración propia.

en todo caso líderes del sector por el que sienten verdadera pasión: deporte, moda y entretenimiento, principalmente. Y es que $63,9 \%$ de los jóvenes reconoció en la encuesta que dentro de su círculo de amistades (following) hay marcas comerciales, empresas u organizaciones (figura 4). 
Para los jóvenes, la red es sinónimo de actualidad e instantaneidad: "Si un tema es tendencia, el interés por participar en el debate y contribuir aumenta frente a otros asuntos" (mujer, 22 años). Aunque si hay una motivación especialmente reseñada para iniciar o secundar un debate, es la de alzar la voz contra abusos o injusticias, principalmente de alcance local.

\section{Un profesorado expectante y cauteloso ante los cambios}

A partir de la consulta a 18 profesores universitarios especialistas en Comunicación y procedentes de cuatro centros diferentes ubicados en Ecuador, se constata que disponen prioritariamente de ordenador fijo y de smartphone, con el consiguiente desembolso económico personal que eso supone. Por el contrario, resulta residual quienes acometen la compra de una tableta o una phablet ${ }^{1}$.Quienes sí lo hacen y se alejan de lo común -el ordenador o el smartphone- reconocen no estar aprovechando todo su potencial, por falta de destreza.

Esta situación refuerza la predisposición del profesorado a mejorar sus habilidades tecnológicas, reclamando a sus centros de trabajo una mayor inversión en formación docente aplicada a las nuevas tecnologías. Sin embargo, la falta de tiempo o los elevados precios de los cursos que les son de interés resultan las limitaciones más señaladas.

El docente, como profesional del ámbito educativo, siente la necesidad y la obligación de permanecer informado de la realidad no solo más cercana y local, sino también de la actualidad nacional e internacional. Principalmente mediante los medios de comunicación tradicionales, el profesor se forja su concepción de lo que sucede, consultándolos prácticamente a diario. Sin embargo, no todos los medios tienen la misma consideración ni se acude a ellos de la misma forma. La prensa digital, por su facilidad de acceso y consulta, resulta la más demandada, al igual que las redes sociales, pues se reconoce su rol en la transmisión de informaciones de última hora, siempre y cuando sean firmadas por periodistas o medios de comunicación constituidos a quienes, además, se les otorga el mismo nivel de credibilidad que a la prensa escrita o digital. No sucede lo mismo con la televisión, que resulta el medio más denostado.

A pesar de confiar en los medios digitales, el profesorado ecuatoriano reconoce que en muchas ocasiones los portales web sucumben al fenómeno del clickbait, ese en el que se ofrece un titular muy llamativo, pero que después no cumple con las expectativas creadas, decepcionando al lector, y cuya única finalidad es la de aumentar las cifras de acceso a la información. En Ecuador, esta forma de proceder se asocia con los contenidos vinculados a la prensa rosa y a los sucesos y, en menor medida, con las secciones de mayor tradición, como la política.

1. Teléfono inteligente con más de 5,5 pulgadas. 
Al profesor universitario le interesan las redes sociales y cree conveniente estar en ellas, aunque reconoce que su presencia es, en la mayoría de los casos, limitada, pues presenta un perfil de baja producción inédita. El miedo a exponerse, a las posibles consecuencias y, sobre todo, al elevado tiempo que demanda esta actividad, emergen como las principales limitaciones al intento por generar contenido propio. Con todo lo expuesto, puede decirse que el uso prioritario que este colectivo aplica a las redes sociales resulta unánime: estar informado. En consecuencia, se trata más de consumir, y no tanto de producir si no es para socializar, es decir, mantener lazos de amistad con otras personas.

Los docentes son conscientes de su interés por sacar el máximo rendimiento a la función informativa de las redes sociales, incorporando a su círculo de amistades o a la lista de a quienes siguen (following) perfiles de periodistas o medios de comunicación en los que se confía. Asimismo, admiten que de forma habitual son viralizadores de los contenidos firmados por dichos medios. El criterio que más pesa para tomar partido e interactuar de alguna forma es que el contenido aborde cuestiones cercanas a los intereses del docente o tenga un poso de denuncia social, y de esta forma se visibilice lo que estos entienden por injusticias. Si bien es cierto que existe un temor a la sobreexposición en redes, los docentes reconocen que los contenidos de temática política, cultural y deportiva son los que les suscitan más ocasiones de comentarios propios en las redes sociales.

\section{CONCLUSIONES}

La eclosión de las redes sociales ha propiciado un cambio en las rutinas productivas, de difusión y de consumo de los medios de comunicación, trastocando la forma de hacer y, en consecuencia, la formación de los futuros periodistas. La capacidad de las redes sociales para transmitir información, aportar visibilidad al trabajo periodístico, interactuar, y ser un espacio de proyección corporativa y de marca personal son razones suficientes para indagar sobre en qué forma asumen esta herramienta y sus soportes los principales colectivos implicados en el proceso de formación: los estudiantes de Comunicación y sus docentes.

Mediante la combinación de varias técnicas - de corte cuantitativo y cualitativose exploraron las rutinas, preferencias y la disposición a participar en las plataformas web de estos colectivos. Los resultados evidencian que los estudiantes universitarios en Comunicación adjudican unos valores y funcionalidades distintas a cada red social, en cierta manera condicionados por su edad y género, lo que explicaría la tendencia generalizada de disponer de un perfil activo en más de tres plataformas. Informarse y, sobre todo, entretenerse, acapararía la mayor parte del tiempo, siendo las redes sociales una fuente de recursos para cubrir necesidades 
tanto emocionales como sociales y relacionales (Del Prete \& Redon, 2020). Los resultados aquí obtenidos señalan que los jóvenes hacen un uso extensivo de las tecnologías, destinado fundamentalmente a relacionarse con sus iguales. También podemos decir que las redes sociales tienen un valor educativo, en el sentido de que forman en valores personales y sociales, tal y como lo señala De Haro (2010). Nuestra investigación detecta la preocupación de los universitarios ecuatorianos por temas sociales, que se manifiesta en el empleo de las redes sociales como espacios de denuncia.

El miedo de los futuros periodistas a ver lastimada su imagen por un comentario o imagen del pasado les preocupa, y eso les lleva a confiar en espacios efímeros cuya marca de identidad es la desaparición de los contenidos publicados transcurridas 24 horas. A pesar de esto, exponen sin pudor aspectos de su vida privada, lo que confirma la H1 de la que partíamos: predisposición a experimentar, interés por la tecnología y carácter irreflexivo, como también lo avalan los trabajos de Caro Samada (2015) o Macedo, Salmeron, Ros, Pérez, Stadtler y Rouet (2020), quienes señalan la incapacidad de los más jóvenes para priorizar fuentes fiables. Asimismo, se comprueba que el smartphone, lejos de ser utilizado como una herramienta facilitadora, se ha convertido en una carga, siendo constantes las reafirmaciones de dependencia y exigencia de uso (Riehm et al., 2019).

Pocos son los referentes del periodismo, activos en el ciberespacio, mencionados por los estudiantes, lo que refuerza la pérdida de liderazgo de los medios (Villafañe, Ortiz, \& Martín, 2020). En contraposición, los jóvenes entrevistados encuentran mayor interés en los contenidos elaborados por instagramers o YouTubers vinculados con las temáticas que les interesan, como la moda, el deporte o el ocio.

En el caso del profesorado, hay un sentimiento generalizado de que Internet y las redes sociales son el presente y el futuro y hay que estar ahí. El esfuerzo por no quedarse atrás es evidente: adquisición de soportes tecnológicos, demanda de formación, autoaprendizaje, etc. Sin embargo, la cautela y sobre todo la necesidad de invertir mucho tiempo - del que no disponen- frenan la creación de contenido propio en las redes sociales. La tendencia mayoritaria es la de controlar con celo quiénes forman parte de su círculo de amistades virtual y dar credibilidad únicamente a las informaciones procedentes de fuentes o medios de comunicación reconocidos, compartiéndolas con frecuencia, aspectos que avalarían la $\mathrm{H} 2$ de partida. Tal y como ocurría con los estudiantes, la finalidad social o de denuncia se presenta como la principal motivación para publicar contenido propio.

Los perfiles analizados evidencian una percepción y uso muy diferente de las redes sociales con base en la edad. De esta manera, los universitarios más jóvenes se apropian de estas para pertenecer a sus modelos de referencia, representados 
por los influencers; los alumnos de posgrado lo asocian con su trabajo profesional, con una alta preocupación por la huella digital, y los profesores exprimen su faceta informativa. En todos los casos, predomina la observación y la viralización de contenidos de otros, tal y como lo concluyen Arribas-Urrutia, Islas-Carmona y Gutiérrez (2019).

Las características en el consumo de los medios han cambiado de manera significativa con la llegada de las redes sociales, tanto en los futuros periodistas como en los hábitos y competencias de sus profesores. El trabajo de Herrero-Diz, Tapia-Frade y Varona-Aramburu (2020) confirma que, en España, la tendencia a consumir noticias a través del smartphone está consolidada, forzando a los cibermedios a ofrecer propuestas móviles atractivas, especialmente en formato vídeo. Esto nos hace pensar en un debate abierto sobre, por un lado, los cambios que requieren los medios de comunicación para recuperar el liderazgo perdido, y por otro, las competencias digitales de los profesores de Periodismo para afrontar una era de convergencia.

\section{REFERENCIAS}

Arias Valencia, M. (1999). Triangulación metodológica: sus principios, alcances y limitaciones (Methodological triangulation: principles, scope, and limits). Investigación y Educación en Enfermería, 18(1), 13-26.

Arribas-Urrutia, A., Islas-Carmona, O., \& Gutiérrez Cortés, F. (2019). De prosumidores a observadores: una tendencia emergente en Internet y en los jóvenes ecuatorianos. Resultados del estudio del World Internet Project, Ecuador (From prosumers to observers: an emergent trend on the Internet and among Young Ecuadorians. Results from the World Internet Project study, Ecuador). Revista Latina de Comunicación Social, (74), 969-996. https://doi.org/10.4185/RLCS-2019-1367

Barrios, A. \& Fajardo, G. C. (2017). El ecosistema educativo universitario impactado por las TIC (The university education ecosystem impacted by ICT). Anagramas. Rumbos y sentidos de la comunicación, 15(30), 101-120. https://doi.org/10.22395/angr.v15n30a5

Bawden, D. (2002). Revisión de los conceptos de alfabetización informacional y alfabetización digital (Information and Digital Literacy: A review of Concepts). Anales De Documentación, 5, 361-408. Retrieved from https://revistas.um.es/analesdoc/article/view/2261

Campos-Freire, F., Rúas-Araújo, J., López-García, X., \& Martínez-Fernández, V. (2016). Impacto de las redes sociales en el periodismo (The impact of social media on journalism). El profesional de la información, 25(3), 449-457. https://doi.org/10.3145/epi.2016.may.15

Caridad, M. \& Martínez, S. (2016). Gobierno y Parlamento abierto: la participación ciudadana en el tratamiento y visualización de la información pública (Government and Open Parliament: Citizens Participation in the Treatment and Visualization of the Public Information). Revista Interamericana de Bibliotecología, 39(1), 47-56. Retrieved from https://revistas.udea.edu.co/index.php/RIB/article/view/25423 
Caro Samada, M. C. (2015). Información y verdad en el uso de las redes sociales por parte de adolescentes (Information and truth in the use of the networks by teenagers). Teoría de la Educación. Revista interuniversitaria, 27(1), 187-199. https://doi.org/10.14201/teoredu2015271187199

Castaño, C. (2006). Alfabetización digital, inclusión y género (Digital literacy, inclusion and gender). In R. Casado (Coord.), Claves de la alfabetización digital (Keys to digital literacy) (pp. 67-76). Madrid, Spain: Fundación Telefónica.

Chaparro Domínguez, M. Á. (2014). Nuevas formas informativas: el periodismo de datos y su enseñanza en el contexto universitario (New information methods: data journalism and its teaching in the university Context). Historia Y Comunicación Social, 19, 43-54. https://doi.org/10.5209/rev_HICS.2014.v19.45009

Cuesta, Ó. J. \& Lugo-Ortiz, L. (2017). Expectativa de los medios de información frente a la formación de periodistas. Análisis comparativo entre Colombia y Puerto Rico (News Media Expectations of Journalism Education. A Comparative Analysis between Colombia and Puerto Rico). Revista Encuentros, 15(1), 15-28. https://doi.org/10.15665/re.v15i1.770

De Haro, J. J. (2010). Redes sociales para la educación (Social networks for education). Madrid, Spain: Anaya.

Del Prete, A. \& Redon, S. (2020). Las redes sociales virtuales: Espacios de socialización y definición de identidad (Virtual social networks: Spaces of socialization and definition of identity). Psicoperspectivas, 19(1), 1-11. https://doi.org/10.5027/psicoperspectivas-vol19-issue1-fulltext-1834

Durán Cuartero, M., Gutiérrez Porlán, I., \& Prendes Espinosa, M. P. (2016). Análisis conceptual de modelos de competencia digital del profesorado universitario (Conceptual analysis of digital competence models of university teacher). RELATEC: Revista Latinoamericana de Tecnología Educativa, 15(1), 97-114. Retrieved from http://hdl.handle.net/10662/5790

Durántez-Stolle, P. (2017). Interacción y participación en salas de prensa virtuales de las empresas del IBEX 35 (Interactive and participatory elements in the virtual press rooms of IBEX 35 companies). El Profesional de la Información, 26(2), 228-237. https://doi.org/10.3145/epi.2017.mar.09

García Orosa, B. \& López García, X. (2016). Las redes sociales como herramienta de distribución on line de la oferta informativa en los medios de España y Portugal (Social networks as a tool for online distributionof information supply media of Spain and Portugal).

Zer. Revista de estudios de comunicación, 21(40), 125-139. Retrieved from https://ojs.ehu.eus/index.php/Zer/article/view/16416

García-Pérez, R., Rebollo-Catalán, Á., \& García-Pérez, F. (2016). Relación entre las preferencias de formación del profesorado y su competencia digital en las redes sociales (The relationship between teacher training preferences and their digital skills on social networks). Bordón. Revista de Pedagogía, 68(2), 137-153. https://doi.org/10.13042/Bordon.2016.68209

García-Ruiz, R., Gozálvez Pérez, V., \& Aguaded Gómez, J. (2014). La competencia mediática como reto para la educomunicación: instrumentos de evaluación (Media competence as a challenge for educommunication: evaluation tools). Cuadernos.Info, (35), 15-27.

https://doi.org/10.7764/cdi.35.623

Gutiérrez, F., Islas, O., \& Arribas, A. (2018). El difícil e indispensable ejercicio del periodismo en América Latina (The difficult and indispensable exercise of journalism in Latin America). In L. Navarro Zamora (Coord.), Miradas del Ciberperiodismo en Iberoamérica (Cyberjournalism in Ibero-America) (pp. 131-152). Editorial Internacional La Latina. 
Herrero-Diz, P., Tapia-Frade, A., \& Varona-Aramburu, D. (2020). La consolidación de los dispositivos móviles para el consumo de información en España (The consolidation of mobile devices for information consumption in Spain). Cuadernos.info, (46), 203-221. https://doi.org/10.7764/cdi.46.1741

Internet World Stats. (2019). Usage and Population Statistics. Retrieved from https://www.internetworldstats.com/south.htm\#ec

Jenkins, H. (2008). Convergence Culture: la cultura de la convergencia de los medios de comunicación (Convergence Culture). Barcelona, Spain: Paidós

Lara, T. (2008). La nueva esfera pública. Los medios de comunicación como redes sociales (The new public sphere. The media as social networks). Telos, 76, 128-131. Retrieved from https://telos.fundaciontelefonica.com/archivo/numero076/los-medios-decomunicacion-como-redes-sociales/?output=pdf

López-García, X., Rodríguez-Vázquez, A. I., \& Pereira-Fariña, X. (2017). Competencias tecnológicas y nuevos perfiles profesionales: desafíos del periodismo actual (Technological Skills and New Professional Profiles: Present Challenges for Journalism). Comunicar, 53, 81-90. https://doi.org/10.3916/C53-2017-08

Lopezosa, C., Codina, L., Díaz-Noci, J., \& Ontalba, J. (2020). SEO y cibermedios: De la empresa a las aulas (SEO and the digital news media: From the workplace to the classroom). Comunicar, 63, 65-75. https://doi.org/10.3916/C63-2020-06

Macedo, M., Salmeron, L., Ros, C., Pérez, A., Stadtler, M., \& Rouet, J. F. (2020). Are frequent users of social network sites good information evaluators? An investigation of adolescents' sourcing abilities. Journal for the Study of Education and Development, 43(1), 101-138. https://doi.org/10.1080/02103702.2019.1690849

Martínez-Costa, M., Serrano-Puche, J., Portilla, I., \& Sánchez-Blanco, C. (2019). La interacción de los jóvenes adultos con las noticias y la publicidad online (Young adults' interaction with online news and advertising). Comunicar, 59, 19-28. https://doi.org/10.3916/C59-2019-02

Martínez-Sanz, R., Islas Carmona, O., Campos Domínguez, E., \& Redondo García, M. (2016). El profesor universitario de Comunicación: acceso, consumo y cultura mediática. Un estudio comparativo entre España y México (Communication professorship: access, consumption and media culture. A comparative study of Spain and Mexico). Revista Latina de Comunicación Social, (71), 349-372. https://doi.org/10.4185/RLCS-2016-1099

Mayoral Sánchez, J. (2016). Ciberperiodismo audiovisual en España: del modelo televisivo a la construcción de una identidad propia (Audiovisual online journalism in Spain: from the television model to build an identity). Zer: Revista de estudios de comunicación, 21(41), 111-129. Retrieved from https://ojs.ehu.eus/index.php/Zer/article/view/17265

Mellado, C. (2011). Examining Professional and Academic Culture in Chilean Journalism and Mass Communication Education. Journalism Studies, 12 (3), 375-391. https://doi.org/10.1080/1461670X.2010.507373

Morse, M. (1991). Approaches to Qualitative-Quantitative Metodological Triangulation. Metodology Corner. Nursing Research, 40(2), 120-123. Retrieved from https://journals. lww.com/nursingresearchonline/Citation/1991/03000/Approaches_to_Qualitative_ Quantitative.14.aspx 
Navarro Zamora, L. (2012). El ciberperiodismo en los móviles como una forma emergente de transmisión de información periodística (Cyberjournalism on mobiles as an emerging form of journalistic information transmission). Question, 1(35), 387-400. Retrieved from https://perio.unlp.edu.ar/ojs/index.php/question/article/view/1579

Negrete-Huelga, K. \& Rivera-Magos, S. (2018). Estrategias de comunicación en redes sociodigitales desde la práctica del gobierno abierto (Communication strategies in social networks from the practice of open government). Cuadernos.Info, (42), 183-196. https://doi.org/10.7764/cdi.42.1242

Nieto Borda, N. (2021). Enseñanza del periodismo transmedia en Colombia, una experiencia pedagógica con estudiantes universitarios. Cuadernos.Info, (48), 215-236.

https://doi.org/10.7764/cdi.48.27827

Noguera, J. M. (2010). Redes sociales como paradigma periodístico. Medios españoles en Facebook (Social networks as journalistic paradigm. Spanish media on Facebook). Revista Latina de Comunicación Social, (65), 176-186. https://doi.org/10.4185/RLCS-65-2010-891-176-186

Noguera, J. M. (2012). Redes y periodismo: cuando las noticias se socializan (Networks and journalism: when the news are socialized). Barcelona, Spain: Editorial UOC.

Odriozola-Chéné, J. \& Rodrigo-Mendizábal, I. (2017). Hacia un periodismo de calidad en Ecuador: perspectivas de periodistas y audiencia (Towards quality journalism in Ecuador: perspectives of journalists and media consumers). Cuadernos.info, (41), 175-192. https://doi.org/10.7764/cdi.41.1100

Picard, R. G. (2014). Twilight or New Dawn of Journalism? Journalism Studies, 15(5), 500-510. https://doi.org/10.1080/17512786.2014.905338

Riehm, K. E., Feder, K. A., Tormohlen, K. N., Crum, R. M., Young, A. S., Green, K. M., Pacek, L. R., La Flair, L. N., \& Mojtabai, R. (2019). Associations between time spent using social media and internalizing and externalizing problems among U.S. youth. JAMA Psychiatry, 76(12), 1266-1273. https://doi.org/10.1001/jamapsychiatry.2019.2325

Salaverría, R. (2010). ¿Ciberperiodismo sin periodistas? Diez ideas para la regeneración de los profesionales de los medios digitales (Cyberjournalism without journalists? Ten ideas for the regeneration of digital media professionals). In F. Campos Freire (Coord.), El cambio mediático (Media change) (pp. 236-249). Zamora, Spain: Comunicación Social Ediciones y Publicaciones.

Sampedro, V. (2015). El cuarto poder en red. Por un periodismo (de código) libre (The fourth power in network. For a free (code) journalism). Quito, Ecuador: Ediciones Ciespal.

Sandí Delgado, J. \& Sanz, C. (2018). Revisión y análisis sobre competencias tecnológicas esperadas en el profesorado en Iberoamérica (Review and analysis of expected technological competences in teachers in Ibero-America). Edutec. Revista Electrónica De Tecnología Educativa, (66), 93-121. https://doi.org/10.21556/edutec.2018.66.1225

Tascón, M. (2012). Guías de uso de las redes sociales para periodistas: entre el afán didáctico y el mecanismo de control. Cuadernos de periodistas: revista de la Asociación de la Prensa de Madrid, (24), 80-87. Retrieved from https://www.apmadrid.es/wp-content/ uploads/2012/06/80-87\%20GUIAS\%20REDES\%20SOCIALES.pdf 
Tejedor, S. \& Cervi, L. (2017). Análisis de los estudios de Periodismo y Comunicación en las principales universidades del mundo. Competencias, objetivos y asignaturas (Analysis of the journalism and communication degree programmes of the world's top-ranked universities. Competencies, objectives and subjects). Revista Latina de Comunicación Social, (72), 1626-1647. https://doi.org/10.4185/RLCS-2017-1238

Túñez-López, J. M., Fieiras Ceide, C., \& Vaz-Álvarez, M. (2021). Impacto de la Inteligencia Artificial en el Periodismo: transformaciones en la empresa, los productos, los contenidos y el perfil profesional (Impact of Artificial Intelligence on Journalism: transformations in the company, products, contents and professional profile). Communication \& Society, 34(1), 177-193. https://doi.org/10.15581/003.34.1.177-193

Villafañe, J., Ortiz, Y., \& Martín, J. L. (2020). Reputación de los medios de comunicación españoles (The reputation of the Spanish media). Profesional de la información, 29(4). https://doi.org/10.3145/epi.2020.jul.07

Yánez, S., Martínez, M., \& Barros, R. (2018). La marca personal, un desafío para los periodistas ecuatorianos (Personal branding, a challenge for the ecuadorian journalists). Tsafiqui Revista Cientifica en Ciencias Sociales, 9(11). https://doi.org/10.29019/tsafiqui.v0i11.491

We are social \& Hootsuite. (2019). Digital Report 2019. Retrieved from https://wearesocial.com/global-digital-report-2019

\section{SOBRE LOS AUTORES}

RAQUEL MARTíNEZ SANZ, es profesora Titular en el área de Periodismo de la Universidad de Valladolid. Miembro del GIR NUTECO, Nuevas Tendencias en Comunicación. Sus principales líneas de trabajo se centran en la comunicación digital y corporativa. Coordinadora del Proyecto de Innovación Docente "Hazte donante", que emplea la educomunicación para el cambio social.

iD https://orcid.org/0000-0002-4753-0282

AMAIA ARRIBAS URRUTIA, es profesora Titular en la Universidad de Los Hemisferios (Ecuador). Directora del Centro de Altos Estudios de Internet y Sociedad de la Información (CAIESI). Directora de Posgrados de la Facultad de Comunicación y Tecnologías de la Información. Directora del proyecto de vinculación "Alfabetización digital". 